\section{Kidney \\ Blood Pressure Research}

\title{
Roles of Non-Coding RNAs in Acute Kidney Injury
}

\author{
Ping Zhou ${ }^{\mathrm{a}}$ Zhi Chen ${ }^{\mathrm{b}}$ Yan Zou ${ }^{\mathrm{a}} \quad$ Xiaoxiao Wan ${ }^{\mathrm{a}}$ \\ aDepartment of Nephrology; 'bntensive Care Unit, Jiangxi Provincial People's Hospital, \\ Nanchang, Jiangxi, China
}

\author{
Key Words \\ microRNA • LncRNA • Acute kidney injury • Ischemia/reperfusion injury • Therapy
}

\begin{abstract}
Acute kidney injury (AKI) is a complex kidney disorder that leads to numerous complications, such as elevated nitrogenous wastes, metabolic acidosis, high potassium levels and even death. The pathogenesis of AKI is very complicated, and its causes are commonly categorized as prerenal, intrinsic or postrenal. In the past few years, evidence has accumulated showing that non-coding RNAs (ncRNAs), such as microRNAs (miRNAs) and long non-coding RNAs (IncRNAs), have significant potential to aid the development of diagnostic and therapeutic strategies of AKI. In this review, we briefly summarize the current understanding of the mechanisms underlying AKI and the main functions of ncRNAs. We mainly focus on revealing the functions of miRNAs (e.g., miR-21, miR-24, miR-30 family, miR-126, miR-127, miR-150, miR-494 and miR-687) and IncRNAs (e.g., TapSAKI, AK139328 and IncRNA-PRINS) in the pathogenesis of AKI.
\end{abstract}

\section{Introduction}

Acute kidney injury (AKI), also known as acute renal failure (ARF), is a common and highly lethal disorder characterized by abrupt deterioration of kidney function that develops within 7 days [1-5]. The pathogenesis of AKI is complicated and generally categorized into prerenal, intrinsic or postrenal causes [6]. Prerenal causes include decreased blood flow in the kidney (e.g., ischemia/reperfusion injury, low blood pressure, blood or fluid loss, heart failure and liver cirrhosis) [6,7]. Intrinsic AKI occurs as a result of damage to the renal tubules, glomeruli or interstitium, which is commonly caused by inflammation or exposure to substances harmful to the kidneys (e.g., acute glomerulonephritis, acute interstitial nephritis, acute tubular necrosis, sepsis, contrast agents and antibiotics) [6, 7]. Finally, postrenal AKI 


\section{Kidney Blood Pressure Research}

is a consequence of urinary tract blockage (e.g., enlarged prostate, a neurogenic bladder of kidney stones) $[6,7]$.

\section{The pathogenesis and mechanisms of acute kidney injury}

In recent decades, great advances have been made in understanding the contributions of apoptosis, endothelial changes and the inflammatory response to AKI [8-11]. After ischemic injury, cells or tissues may undergo alterations in a number of characteristics, such as morphology (e.g., loss of proximal tubule brush border and tubule cells, pathological changes of proximal tubular dilation and distal tubular casts) [2], hemodynamics (e.g., disruption of blood flow patterns and exchange of oxygen) [2], tubule dynamics and cell metabolism (e.g., adenine nucleotide, intracellular calcium and reactive oxygen molecules) [2], tubule cell structure (apical cytoskeleton, basolateral cytoskeleton and cell viability) [2], microvasculature and inflammatory responses, as well as changes in global gene expression [2]. Of these, alterations in tubule cell metabolism, tubule cell structure, the microvasculature and inflammation have been well studied [2]. In the case of alterations in tubule cell metabolism, the initiation phase of AKI is commonly associated with ATP depletion, which results in the activation of a number of oxidative and cell death pathways $[2,8,12]$. These signaling pathways are completed during the extension phase through prolonged ischemia followed by reperfusion, causing cell apoptosis and oxidant injury $[8,13]$. For alterations in tubule cell structure, cells experiencing loss of brush borders, disrupted polarity and disrupted cytoskeletal structure in the initiation phase of AKI may progress to the extension phase, which can cause cell death, luminal obstruction, desquamation and inflammatory responses if the injury is not alleviated $[2,8]$. In terms of alterations in the microvasculature and inflammation, injury of endothelial cells leads to drastic vasoconstriction, microvasculature sludge and congestion of the microvasculature with leukocytes [2, 8]. Activated leukocytes lead to the activation of many inflammatory mediators and the production of reactive oxygen species (ROS) that enhance tubule cell damage $[8,11,13]$.

In addition, overwhelming evidence now indicates that apoptosis is a pathogenic mechanism of AKI $[12,14,15]$. As shown in Figure 1, in the extrinsic pathway, the cell surface death receptor Fas stimulates the formation of a multiprotein complex termed the death-inducing signaling complex (DISC) [16], resulting in the activation of procaspase-8. The activated caspase-8 further cleaves downstream effector caspases, such as caspase-3, leading to apoptosis [16]. In the intrinsic pathway, the intracellular death signal is transmitted to the mitochondria through a number of proteins including p53, Puma (p53 upregulated modulator of apoptosis) and the B-cell lymphoma-2 (Bcl-2) protein family. Bax and Bak stimulate the release of cytochrome $c$, which forms the apoptosome with Apaf- 1 and caspase-9, resulting in the activation of caspase-3 and triggering apoptosis (Figure 1) [16]. All of these major apoptotic pathways are activated in the process of ischemic AKI [12, 14, 15]. Inhibition of these pathways is a promising avenue for the treatment of AKI.

\section{Non-coding RNAs and their main functions in disease}

Non-coding RNAs (ncRNAs) represent a diverse family of regulatory transcripts that are transcribed from DNA but not translated into proteins [17-19]. Based on different properties, ncRNAs can be categorized into different classes. For instance, ncRNAs can be classified as microRNAs (miRNAs) ( $\sim 22 \mathrm{nt}$ ) or long non-coding RNAs (lncRNAs) ( $>200 \mathrm{nt}$ ) based on their length [20-22]. In addition, ncRNAs can also be classified as ribosomal (rRNAs) or transfer RNAs (tRNAs), as well as small nuclear RNAs (snRNAs), small nucleolar RNAs (snoRNAs), cytoplasm-located piwi-interacting RNAs (piRNAs) or short interfering RNAs (siRNAs) based on their function or subcellular localization [23]. However, it is difficult to clearly separate categories of ncRNAs because many often share multiple properties [23].

It has been well demonstrated that miRNAs regulate a wide range of biological processes including cell development, differentiation, proliferation, metabolism and apoptosis [24, 


\section{Kidney Blood Pressure Research}

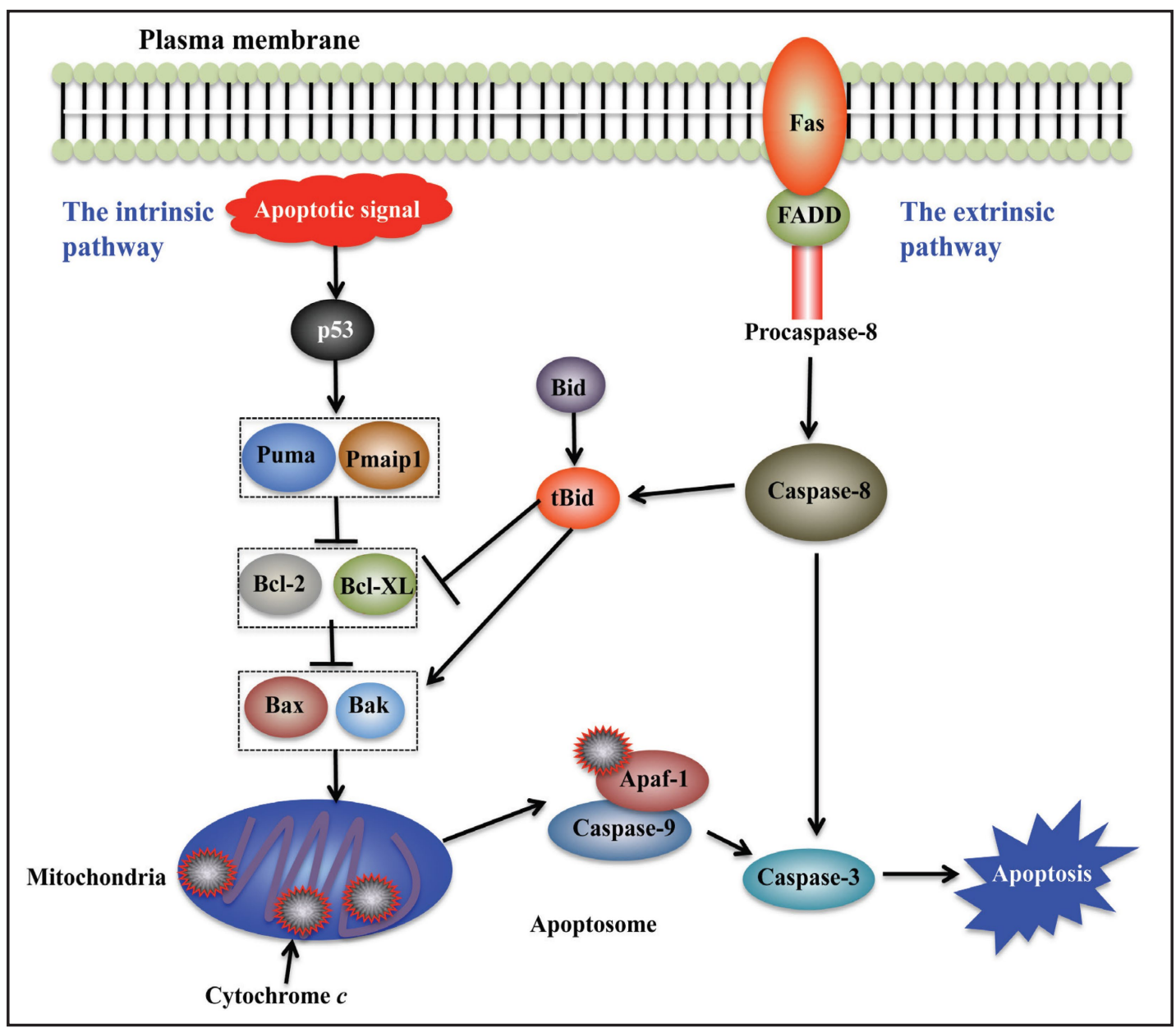

Fig. 1. Major apoptotic pathways involved in AKI. Apoptosis is mediated by two main pathways: the mitochondrial pathway (intrinsic) and the cell death receptor-mediated pathway (extrinsic) [16]. The intrinsic pathway is initiated intracellularly, and pro-apoptotic proteins are released from the mitochondria to activate caspases and trigger apoptosis [16]. The extrinsic pathway is mainly mediated through the Fas pathway [16]. The stimulation of cell surface death receptor Fas leads to receptor aggregation and the recruitment of the adaptor molecule FADD and procaspase-8, which subsequently becomes activated and initiates apoptosis by direct cleavage of downstream effector caspases [16]. Puma, p53 upregulated modulator of apoptosis; PMAIP1, Phorbol-12-myristate-13-acetate-induced protein 1, also known as Noxa; Apaf-1, apoptotic protease activating factor-1; FADD, Fas-associated death domain; Bcl-2, B-cell lymphoma-2; Bid, BH3 interacting domain death agonist.

25]. A significant number of studies have revealed that dysregulation of individual miRNAs or subsets is associated with the pathogenesis of multiple human disease, such as cancer, cardiovascular disease, cardiac failure, inflammatory diseases, neurodevelopmental diseases, autoimmune diseases, viral infections and metabolic diseases $[25,26]$. The primary role of miRNAs in these diseases is gene regulation at the transcriptional or post-transcriptional level $[25,26]$. The expression of miRNAs can be altered by many factors, including chromosomal amplification or deletion, modifications in promoter region and activation of transcription factors [27-29]. miRNA functions can be broadly classified into two categories: (i) homeostatic regulation of gene expression and (ii) regulation of the robustness of cellular responses [27-29].

Emergingstudies indicate that lncRNAsare critical in a broad range of biological processes and are associated with a number of diseases, such as cancer, cardiovascular diseases and 


\section{Kidney Blood Pressure Research}

Table 1. miRNAs and their expression during AKI or I/R injury

\begin{tabular}{|c|c|c|c|c|}
\hline miRNAs & Targets & Biological Role & Species & References \\
\hline miR-21 & $\begin{array}{l}\text { PDCD4, FasL, PTEN, } \\
\text { MyD88, IRAK1 and } \\
\text { PPAR- } \alpha\end{array}$ & $\begin{array}{l}\text { Involvement in apoptosis, } \\
\text { inflammation and fibrosis }\end{array}$ & Human & {$[33,45]$} \\
\hline $\operatorname{miR}-24$ & S1PR1, H2AFX, HO-1 & $\begin{array}{l}\text { Stimulates apoptosis in endothelial } \\
\text { and tubular epithelial cells }\end{array}$ & $\begin{array}{l}\text { Human, } \\
\text { mice }\end{array}$ & {$[34]$} \\
\hline $\begin{array}{l}\text { miR-30 } \\
\text { family }\end{array}$ & Unknown & Unknown & $\begin{array}{l}\text { Human, } \\
\text { rat }\end{array}$ & {$[35]$} \\
\hline $\operatorname{miR}-126$ & CXCR4, SDF1 & $\begin{array}{l}\text { Facilitates vascular regeneration } \\
\text { and supports kidney recovery }\end{array}$ & Mice & [53] \\
\hline $\operatorname{miR}-127$ & KIF3B & $\begin{array}{l}\text { Promotes focal adhesion complex } \\
\text { assembly, cell adhesion and the } \\
\text { integrity of tight junctions }\end{array}$ & Rat & {$[37]$} \\
\hline miR-150 & IGF-1R & $\begin{array}{l}\text { Associated with the suppression of } \\
\text { inflammation and interstitial cell } \\
\text { apoptosis }\end{array}$ & Mice & [38] \\
\hline $\operatorname{miR}-494$ & ATF3 & $\begin{array}{l}\text { Contributes to apoptosis and } \\
\text { inflammation }\end{array}$ & Mice & [39] \\
\hline $\operatorname{miR}-687$ & PTEN & $\begin{array}{l}\text { Facilitates cell cycle progression and } \\
\text { apoptosis that mediates kidney } \\
\text { injury and repair }\end{array}$ & Mice & [40] \\
\hline
\end{tabular}

neurodegeneration diseases $[19,30]$. Generally, lncRNAs regulate gene transcription, induce chromatin remodeling and modification, modulate alternative splicing patterns, serve as scaffolds for higher-order complexes and function as small RNA precursors [19].

\section{miRNAs and AKI}

Current evidence has revealed that miRNAs can function as negative regulators of gene expression in the initiation and/or progression stages of kidney disease [31,32]. As shown in Table 1, a number of miRNAs, such as miR-21 [33], miR-24 [34], miR-30 family [35], miR126 [36], miR-127 [37], miR-150 [38], miR-494 [39] and miR-687 [40], have been implicated in both protective and pathogenic roles in the development of AKI.

miR-21

Renal ischemia/reperfusion (I/R) injury, a major cause of AKI, is associated with significant morbidity and mortality $[41,42]$. The pathogenesis of renal I/R injury and its related molecular mechanisms are extremely complicated; however, much of the damage during reperfusion is mediated by ROS $[11,13]$. ROS-activated inflammatory cells can cause the release of interleukin (IL), tissue necrosis factor (TNF) and other inflammatory factors and can also stimulate expression of hypoxia-inducible factor-1 (HIF-1), thereby promoting cell apoptosis and increasing tissue damage during reperfusion $[11,13]$. In studies evaluating global miRNA expression profiles in renal I/R injury models, several miRNAs, including miR-20a, miR-21, miR-146a, miR-187, miR-192, miR-194, miR-199a-3p, miR-214 and miR805 , are differentially expressed upon I/R injury when compared to controls [43]. Of these miRNAs, the role of miR-21 in renal I/R injury has been widely studied and found to be involved in multiple pathophysiological processes including inflammation, fibrosis and cell survival during I/R injury $[33,44]$.

The inflammatory response is initiated quickly after I/R injury, and this process is associated with the up-regulation of miR-21, which provides protection against the initiation of apoptosis by targeting programmed cell death 4 (PDCD4), Fas ligand (FasL) and phosphatase and tensin homology (PTEN) (Figure 2A) [44]. Meanwhile, inflammation may also be reduced as a result of miR-21 targeting of peroxisome proliferator activated receptor- $\alpha$ (PPAR- $\alpha$ ) and a decrease in NF- $\kappa B$-induced inflammation (Figure 2B) [45]. In addition, miR-21 contributes to Xenon-conferred amelioration of renal I/R injury in mice 


\section{Kidney Blood Pressure Research}

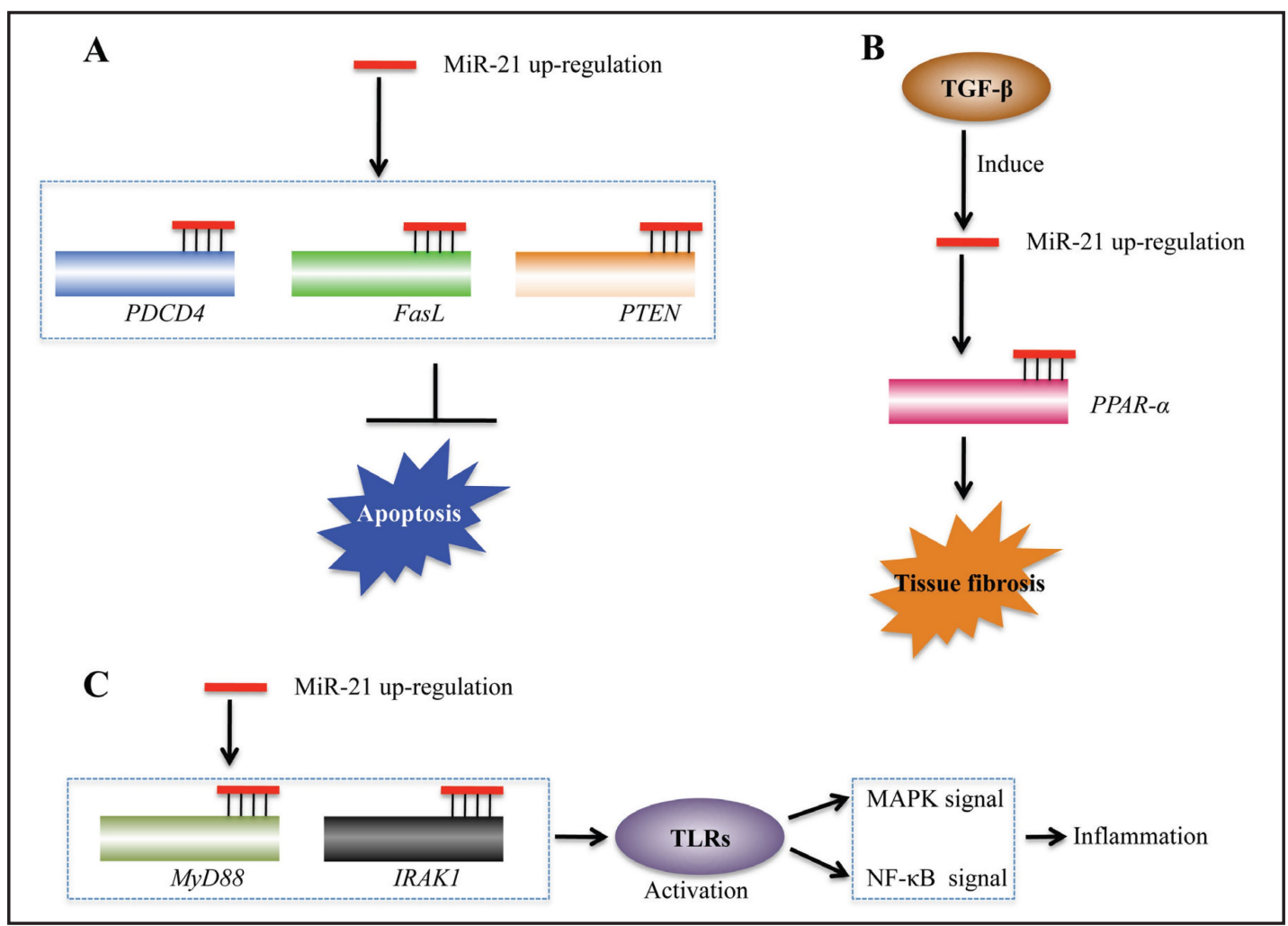

Fig. 2. The different roles of miR-21 in AKI. (A) Up-regulation of miR-21 provides protection against apoptosis by targeting PDCD4, FasL and PTEN. (B) TGF- $\beta$ can upregulate miR-21 expression, which further targets PPAR- $\alpha$ to exacerbate tissue fibrosis. (C) Up-regulation of miR-21 can target MyD88 and IRAK1 and result in the activation of TLRs, which further activates phosphorylation and ubiquitination events and triggers MAPK and NF- $\kappa$ B signals to produce proinflammatory cytokines.

through the regulation of PDCD4, PTEN, HIF-1 $\alpha$ and the PTEN-AKT/MAPK3/VEGF pathway (PTEN-AKT, protein kinase B; MAPK3, mitogen-activated protein kinase 3; VEGF, vascular endothelial growth factor) [44, 46]. Moreover, excessive up-regulation of miR-21 induced by transforming growth factor TGF- $\beta$ stimulation exacerbates tissue fibrosis via suppression of PPAR- $\alpha$ or sprouty homolog 1 (Spry1), resulting in chronic kidney injury [44]. In the end stage of AKI, overexpression of miR-21 can target myeloid differentiation primary response 88 (MyD88) and interleukin-1 receptor-associated kinase 1 (IRAK1), leading to the continuous activation of certain Toll-like receptors (TLRs), which further amplifies phosphorylation and ubiquitination cascades and triggers NF- $\kappa \mathrm{B}$ to produce pro-inflammatory cytokines (Figure 2C) [33]. The diverse functions of miR-21 in I/R injury-associated processes may result from changes in temporal expression of miR-21 target genes and pathways involved in different stages of I/R pathology [44].

Recently, in a septic AKI mouse model, it was found that xenon preconditioning could protect against lipopolysaccharide-induced AKI via regulation of signaling pathways targeted by miR-21 [47, 48]. In fact, xenon preconditioning caused upregulation of miR-21. This upregulation was shown to be relevant in the renal injury and repair process via suppression of the target effector PTEN, which resulted in the upregulation of phosphorylated-Akt (p-Akt) and antiapoptotic Bcl-2 and a decrease in caspase- 3 activity, thereby inhibiting renal cell apoptosis and attenuating LPS-induced AKI $[47,48]$.

$\operatorname{miR}-24$

In a CD-1 mouse model, deletion of DICER, an endoribonuclease that cleaves doublestranded RNA, in the glomerulus resulted in a progressive loss of podocyte function and led 


\section{Kidney \\ Blood Pressure Research}

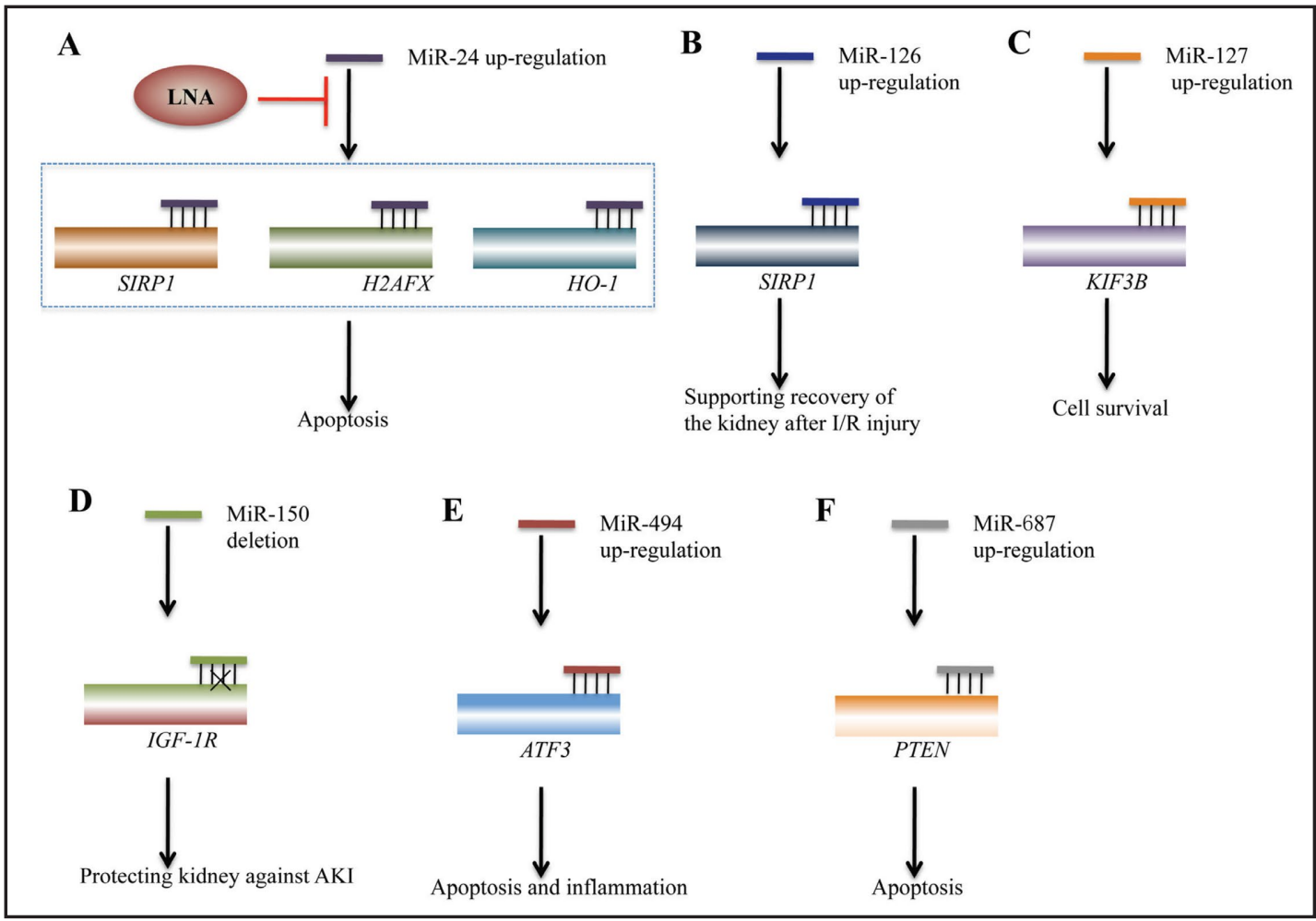

Fig. 3. miRNAs target different genes in AKI. (A) Overexpression of miR-24 targets antiapoptotic proteins, such as S1PR1, H2AFX and H0-1, to trigger apoptosis in endothelial and tubular epithelial cells. (B) Overexpression of miR-126 results in attenuated expression of $C X C R 4$, thereby supporting recovery of the kidneys after I/R injury. (C) Overexpression of miR-127 protects PT cells against I/R injury by targeting KIF3B. (D) Deletion of miR-150 protects the kidneys against AKI by targeting IGF-1R. (E) Overexpression of miR-494 targets ATF3, contributing to inflammation after I/R injury. (F) Overexpression of miR-687 targets PTEN, facilitating cell cycle progression and apoptosis.

to proteinuria and kidney function impairment $[49,50]$. Three microRNAs, including miR$23 \mathrm{~b}, \mathrm{miR}-24$ and miR-26a, have been identified as contributors to this renal failure $[49,50]$.

Recently, miR-24 was shown to play an important role in the process of I/R injury by stimulating apoptosis in endothelial and tubular epithelial cells [34]. Expression of miR-24 was significantly elevated in the kidneys of mice after I/R injury and in patients after kidney transplantation [34, 51]. Cell-sorting experiments after I/R injury in mice revealed that miR-24 is specifically up-regulated in renal endothelial and tubular epithelial cells [34]. In vitro studies suggested that anoxia/hypoxia could induce elevated expression of miR-24 in endothelial and tubular epithelial cells by targeting prominent antiapoptotic proteins, such as sphingosine-1-phosphate receptor 1 (S1PR1), H2A histone family, member X (H2AFX) and heme oxygenase 1 (HO-1) (Figure 3A) [34]. Interestingly, overexpression of these miR-24 targets lacking miR-24 binding sites alongside miR-24 precursors rescued miR-24mediated apoptosis and impaired tube formation and migration capacities [34]. Conversely, silencing of miR-24 in vivo alleviated renal I/R injury, reduced the rate of apoptosis and led to a significant improvement in survival and kidney function [34]. Moreover, treatment with a locked nucleic acid (LNA) that targeted miR-24 improved renal outcomes and survival in mice subjected to bilateral I/R injury [34]. Therefore, inhibition of miR-24 is a promising therapeutic option for patients with ischemic AKI. 


\section{Kidney Blood Pressure Research}

Kidney Blood Press Res 2016;41:757-769

DOI: 10.1159/000450566

Published online: November 11, 2016

(C) 2016 The Author(s). Published by S. Karger AG, Base

www.karger.com/kbr

Zhou et al.: Non-Coding RNAs and Acute Kidney Injury

miR-30 family

Recently, in a contrast-induced rat AKI model, Gutiérrez-Escolano et al. used_miRNA microarray assays to detect miRNAs in rat kidney tissues [35]. They found that 51 miRNAs were aberrantly expressed, and one third of these miRNAs (17) showed a $>2$-fold change in expression in the kidney tissues of contrast-induced AKI rats [35]. Among these 17 miRNAs, the levels of three miR-30 family members (miR-30a, miR-30c and miR-30e), as well as miR320 , were significantly increased in the plasma of contrast-induced AKI rats, while let-7a and miR-200a were significantly decreased [35]. Similar results were also obtained in a validation study with human plasma samples from contrast-induced AKI patients [35]. These results indicated that three miR-30 family members (miR-30a, miR-30c and miR-30e) could serve as biomarkers for contrast-induced AKI detection [35]. However, no targets of these miRNAs in contrast-induced AKI rats or patients have been reported. In an earlier study, Agrawal et al. found that the miR-30 miRNA family (miR-30a-5p, miR-30b, miR-30c-1, miR-30c-2, miR$30 \mathrm{~d}$ and miR-30e) regulates Xenopus pronephros development and targets the transcription factor Lhx1 (LIM-class homeobox factor 1) [52], which may provide valuable insight for further study of the mechanisms of the miR-30 family.

miR-126

The integrity of the peritubular capillary plexus is thought to be important for recovery from I/R injury [53]. In a mouse model, hematopoietic overexpression of miR-126 led to attenuated expression of C-X-C chemokine receptor type 4 (CXCR4) in bone marrow-derived Lin(-)/Sca-1(+)/cKit(+) cells and increased expression of ligand stromal cell-derived factor 1 (SDF-1), thereby facilitating vascular regeneration and supporting kidney recovery after I/R injury (Figure 3B) [53]. This process increased the number of bone marrow-derived end othelial cells and was associated with a higher density of the peritubular capillary network in the corticomedullary junction [53]. Silencing of miR-126 could increase expression of SDF-1 after I/R injury, which resulted in the mobilization of vascular progenitor cells into circulation [55]. Moreover, analysis of cell lysates from whole kidneys revealed that elevated expression of VEGF-A (vascular endothelial growth factor A) and NOS3 (nitric oxide synthase 3 ) after I/R injury resulted from systemic silencing of miR-126 [53].

$\operatorname{miR}-127$

Microarrays and RT-PCR analysis in proximal tubule (PT) cells and in a rat renal I/R model have shown that expression of miR-127 is induced during I/R injury [37]. Subsequent analysis indicated that miR-127 could protect PT cells against I/R injury by targeting kinesin family member 3B (KIF3B) (Figure 3C), a protein involved in cell trafficking [37]. In vitro interference approaches demonstrated that HIF- $1 \alpha$ regulates miR-127 in HK-2 cells in response to hypoxia/reoxygenation (H/R), although the binding site of HIF- $1 \alpha$ has not been successfully identified [37]. Moreover, overexpression of miR-127 promoted focal adhesion complex assembly, cell adhesion and the integrity of tight junctions during $H / R$, implying that it has an important role in the maintenance of cell-matrix and cell-cell adhesion $[37,55]$. These results demonstrate that both miR-127 and KIF3B are critical mediators of proximal epithelial tubule cell responses to I/R and may have potential future applications in the management of renal ischemic damage [37].

$\operatorname{miR}-150$

Previously, miR-150 has been identified to promote renal fibrosis in lupus nephritis through targeting suppressor of cytokine signaling 1 (SOCS1) [56]. Recently, in a mouse model of myocardial infarction (MI)-induced AKI, which mimics human cardiac bypass surgery, deletion of miR-150 protected the kidneys against AKI and bilateral renal I/R injury and was also associated with the suppression of inflammation and interstitial cell apoptosis [38]. When subjecting this mouse model to I/R injury, deletion of miR-150 caused significantly decreased levels of serum creatinine in comparison to WT mice [38]. Immunofluorescence staining with an endothelial marker (cluster of differentiation 31-CD31) and an apoptosis marker (TdT-mediated dUTP nick end labeling-TUNEL) suggested that endothelial cells, but 


\section{Kidney Blood Pressure Research}

Kidney Blood Press Res 2016;41:757-769

DOI: 10.1159/000450566

Published online: November 11, 2016

(c) 2016 The Author(s). Published by S. Karger AG, Base

www.karger.com/kbr

Zhou et al.: Non-Coding RNAs and Acute Kidney Injury

not epithelial cells, undergo apoptosis [38]. Moreover, expression of urinary biomarkers, including kidney injury molecule-1 (KIM-1) and neutrophil gelatinase-associated lipocalin (NGAL), as well as inflammatory cytokines, was significantly reduced in miR-150 knockout mice compared to WT after I/R injury [38]. In addition, miR-150 deletion induced expression of insulin-like growth factor-1 receptor (IGF-1R), one of its target genes (Figure 3D). Conversely, overexpression of miR-150 in endothelial cells caused reduction of IGF-1R [38]. These results indicate that miR-150 mediates its detrimental effects via suppression of the IGF-1R signaling pathways. In addition, a miR-150 deficiency is also able to prevent Fasinduced hepatocyte apoptosis and acute liver injury through regulation of the Akt signaling pathway [57].

\section{miR-494}

miR-494 was initially shown to be upregulated in Waldenstrom macroglobulinemia cells and human retinoblastoma tissues [58]. In a mouse model, miR-494 was shown to target the 3'-UTR of activating transcription factor 3 (ATF3) [39], a protein that has been shown to protect against renal I/R injury [59]. This specific binding represses the transcription of ATF3, thereby contributing to inflammation after I/R injury (Figure 3E) [39]. In this study, overexpression of miR-494 significantly decreased ATF3 expression after renal I/R injury but induced inflammatory mediators, such as IL-6, monocyte chemotactic protein-1 (MCP-1) and P-selectin, thereby exacerbating apoptosis and further decreasing renal function [39]. In addition, overexpression of miR-494 resulted in a significant increase in urinary serum creatinine levels [39]. Similarly, increased miR-494 levels were also found in human patients with AKI [39]. Activation of NF- $\kappa B$ was suggested to mediate this pro-inflammatory response [39]. Inhibition of miR-494 was able to increase neovascularization and blood flow recovery after ischemia likely through targeting of fibroblast growth factor receptor 2 (FGFR2), vascular endothelial growth factor A (VEGFA) and Ephrin B2 (EFNB2) [60].

\section{miR-687}

Microarray assays of kidney tissues from renal ischemia mice identified miR-687 upregulation as transient, with the highest upregulation detected at 12 hours of reperfusion [40]. Sequence analysis and in vitro and in vivo studies indicated that miR-687 is a direct transcriptional target of HIF-1 during renal I/R injury (Figure 3F), and their binding is dependent on a hypoxia responsive element (HRE) [40,61]. MiR-687 also targets PTEN and represses its expression, thereby facilitating cell cycle progression and apoptosis and eventually mediating kidney injury and repair [40]. Inhibition of miR-687 via anti-miR-687 preserved PTEN expression and blocked cell cycle activation and renal apoptosis, leading to protection against kidney injury in mice [40]. These results suggest the possibility of targeting the HIF-1/miR-687/PTEN signaling pathway during I/R injury in future therapeutic strategies [40].

\section{Other miRNAs}

In addition to the above-mentioned miRNAs, a large number of miRNAs are also aberrantly expressed in animal AKI models and human AKI patients. For example, miR-92b, miR-210, miR-617, miR-638 and miR-663b were highly expressed in the plasma of patients with AKI compared to healthy controls [62], whereas let-7b, let-7f, miR-16, miR-320, miR620 and miR-1244 were significantly depressed in the same test samples [62]. Of these miRNAs, only miR-16 was recently found to transactivate via the CCAAT enhancer binding protein beta (C/EBP- $\beta$ ), thereby resulting in aggravated I/R-induced AKI [63]. In addition, as mentioned earlier, in a contrast-induced rat AKI model [35], a many miRNAs, including miR-10a, miR30a, miR30c, miR30e, miR-181, miR-320, miR-455, miR-489 and miR-505, were upregulated, while some miRNAs, including let-7c, miR-146, miR-182, miR-200a, miR200c, miR-204 and miR-302, were downregulated [35]. However, all of these miRNAs have not been well studied in AKI animal models and human patients. It is still unclear which sequences are targeted by each miRNA and how these miRNAs contribute to AKI. 


\section{Kidney Blood Pressure Research}

Table 2. IncRNAs and their expression in AKI or I/R injury

\begin{tabular}{lllcc}
\hline IncRNAs & Targets & \multicolumn{1}{c}{ Biological Role } & Species & References \\
\hline TapSAKI & Unknown & Unknown & Human & {$[64]$} \\
AK139328 & Unknown & $\begin{array}{l}\text { Involvement in apoptosis, } \\
\text { inflammation }\end{array}$ & Mouse & {$[65]$} \\
lncRNA-PRINS & RANTES & $\begin{array}{l}\text { Involvement in inflammation } \\
\text { lno }\end{array}$ & Mice & {$[66]$} \\
\hline
\end{tabular}

\section{Long non-coding RNAs and AKI}

Emerging evidence shows that lncRNAs are critical regulators of genes involved in a variety of human diseases including AKI [64-66]. Recently, microarray assays and RNAsequencing (RNA-seq) methods have been used to evaluate lncRNA expression in animal models of I/R injury or in patients with AKI [64-66]. A few lncRNAs are differentially expressed in models of I/R injury in comparison to the control group expression [64-66]. Of these lncRNAs, the functions of TapSAKI (TrAnscript Predicting Survival in AKI) [64], AK139328 [65] and IncRNA-PRINS (Psoriasis susceptibility-related RNA Gene Induced by Stress) have been well studied (Table 2) [66].

\section{TapSAKI}

In a study evaluating the pattern of circulating lncRNAs in patients with AKI, a genomewide expression analysis was performed using RNA isolated from kidney biopsy and plasma samples of 109 AKI patients, 30 age-matched healthy controls and 30 patient controls with acute myocardial infarction (AMI) [64]. An intronic (antisense to $\beta$-1, 4-mannosylglycoprotein $4-\beta-N$-acetylglucosaminyltransferase-MGAT3) lncRNA termed TapSAKI was specifically identified in kidney biopsies and upregulated in the plasma of patients with AKI but not in the plasma of control group patients [64]. The concentration of circulating TapSAKI correlated with parameters of disease severity and was enriched in hypoxic tubular epithelial cells [64]. These results suggest that TapSAKI may be useful as a specific, independent biomarker for the prognosis of AKI [64]. However, the underlying mechanisms of TapSAKI induction and regulation are still unknown.

\section{AK139328}

Microarray analysis identified a total of 98 lncRNAs that were dysregulated in mouse livers after I/R treatment [65]. Of them, 71 lncRNAs were upregulated and 27 were downregulated [65]. Among the upregulated lncRNAs, AK139328 was shown to be involved in liver I/R injury and exhibited the highest expression levels in normal mouse livers [65]. siRNA-mediated knockdown of AK139328 repressed expression of IP-10 (interferon gamma-induced protein 10) and $M C P-1$, decreased caspase-3 activation and plasma aminotransferase activities and inhibited NF- $\kappa$ B activity and the expression of inflammatory cytokines (Figure 4A) [65]. Increased levels of some survival-signaling proteins, such as glycogen synthase kinase 3 (pGSK3), phosphorylated Akt (pAkt) and endothelial nitric oxide synthase (peNOS), have been detected following knockdown of AK139328, indicating that its removal can activate the Akt signaling pathway (Figure 4A) [65]. Thus, AK139328 may be useful as a biomarker for diagnosis and may serve as a target for improving outcomes after liver surgery or transplantation [65].

\section{InCRNA-PRINS}

RANTES (regulated on activation, normal T-cell expressed and secreted) is an inflammatory mediator involved in I/R-related diseases including AKI; increased RANTES levels in renal tubular cells further aggravated kidney injury through recruitment of inflammatory cells and led to a loss of renal function after I/R injury [66]. Expression of RANTES was significantly increased in wild type mice with I/R injury [66]. Interestingly, RANTES knockout (RANTES(-/-) mice displayed better renal function because their serum creatinine levels were significantly lower than those of wild type mice [66]. Moreover, 


\section{Kidney Blood Pressure Research}

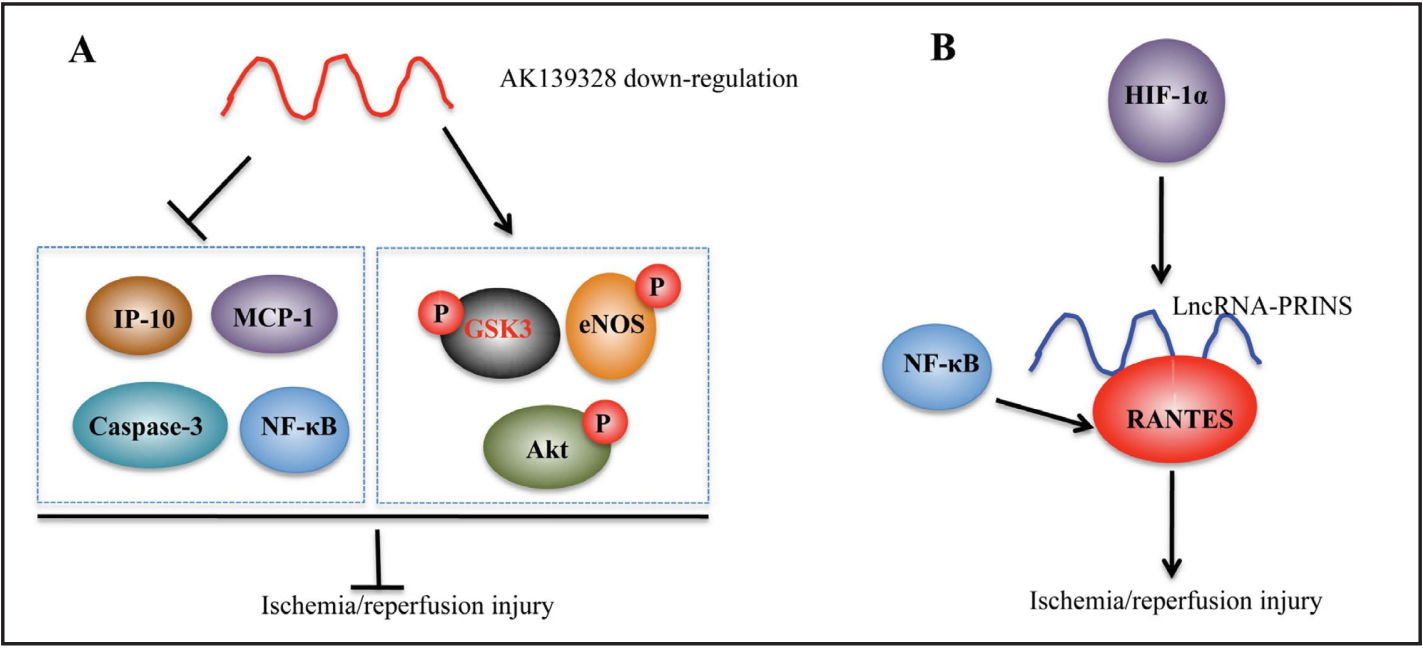

Fig. 4. Involvement of IncRNAs in ischemia/reperfusion injury. (A) Silencing of AK139328 represses expression of $I P-10$ and $M C P-1$, decreases caspase- 3 activation and inhibits NF- $\kappa$ B activity. This silencing can also activate the Akt signaling pathway by increasing levels of phosphorylated GSK3, Akt and eNOS, resulting in attenuation of ischemia/reperfusion injury.

RANTES $^{(-/-)}$mice exhibited defects, such as decreased acute tubular necrosis (ATN), impaired infiltration of inflammatory cells and attenuated proinflammatory production of cytokines and chemokines [66]. These results demonstrate that RANTES is an important mediator of AKI following I/R injury [66]. A HIF-1 $\alpha$-responsive lncRNA known as lncRNA-PRINS has been implicated in regulating RANTES production and may be involved in the process of AKI [66]. Furthermore, RANTES expression is regulated by NF- $\kappa \mathrm{B}$, and lncRNA-PRINS may act as a HIF-1 $\alpha$-dependent lncRNA during AKI (Figure 4B) [66]. Thus, the HIF-1 $\alpha$-IncRNA-PRINSRANTES axis may play a regulatory role during AKI [66].

\section{Conclusions}

In recent years, a great number of ncRNAs have been identified and shown to be critical regulators of human diseases including AKI. We have also made considerable strides in understanding how ncRNAs contribute to kidney function, especially with regard to AKI. Overall, the identified ncRNAs mainly function to target and repress expression of genes that are critical in the development and progression of renal diseases. Meanwhile, multiple signaling pathways involved in apoptosis and inflammation are also dysregulated during AKI. It is highly possible that we will be able to develop future therapeutic strategies that target ncRNAs in vivo to inhibit or restore their expression. Some of these ncRNAs have the potential to be developed as novel diagnostic and prognostic biomarkers for AKI. However, several challenges remain for experimental studies and clinical therapy. The first challenge is that detection and amplification of ncRNAs in the kidneys is challenging because of their low abundance, which may result in key ncRNAs being overlooked during experimental studies. The second challenge is that ncRNAs commonly have cell-specific effects, which poses significant challenges, especially in targeting ncRNA-based therapeutics to specific renal cells. The third challenge is that it is difficult to obtain sufficient clinical samples from patients undergoing different stages of AKI, which may limit our ability to study how ncRNAs function in the process of AKI. Although these challenges exist, understanding the molecular mechanisms of ncRNAs in AKI will have significant benefits for the development of diagnostic and therapeutic tools for early detection and subsequent treatment of AKI. 


\section{Kidney \\ Blood Pressure Research}

Disclosure Statement
Kidney Blood Press Res 2016;41:757-769

\begin{tabular}{|l|l|}
\hline DOI: 10.1159/000450566 & ( 2016 The Author(s). Published by S. Karger AG, Basel
\end{tabular} Published online: November 11, 2016 www.karger.com/kbr

Zhou et al.: Non-Coding RNAs and Acute Kidney Injury

The authors declare no conflicts of interest. The authors did not perform any of the animal or human studies discussed in this article.

\section{Acknowledgments}

We apologize to all authors whose contributions were not cited due to space limitations.

\section{References}

1 Angeli P, Rodriguez E, Piano S, Ariza X, Morando F, Sola E, Romano A, Garcia E, Pavesi M, Risso A, Gerbes A, Willars C, Bernardi M, Arroyo V, Gines P: Acute kidney injury and acute-on-chronic liver failure classifications in prognosis assessment of patients with acute decompensation of cirrhosis. Gut 2015;64:1616-1622.

2 Belardi JA, Albertal M: Elevated biomarkers and contrast-induced acute kidney failure: what comes first the chicken or the egg? Catheter Cardiovasc Interv 2015;85:343-344.

3 Correa RR, Pucci KR, Rocha LP, Pereira Junior CD, Helmo FR, Machado JR, Rocha LB, Rodrigues AR, Gloria MA, Guimaraes CS, Camara NO, Reis MA: Acute kidney injury and progression of renal failure after fetal programming in the offspring of diabetic rats. Pediatr Res 2015;77:440-446.

4 Dirkes S: Acute kidney injury: not just acute renal failure anymore? Crit Care Nurse 2011;31:37-49.

$5 \quad$ Maiwall R, Sarin SK, Moreau R: Acute kidney injury in acute on chronic liver failure. Hepatol Int 2016;10:245-257.

6 Basile DP, Anderson MD, Sutton TA: Pathophysiology of acute kidney injury. Compr Physiol 2012;2:13031353.

7 Koza Y: Acute kidney injury: current concepts and new insights. J Inj Violence Res 2016;8:58-62.

8 Devarajan P: Update on mechanisms of ischemic acute kidney injury. J Am Soc Nephrol 2006;17:15031520.

9 Bonventre JV, Yang L: Cellular pathophysiology of ischemic acute kidney injury. J Clin Invest 2011;121:4210-4221.

10 Sharfuddin AA, Molitoris BA: Pathophysiology of ischemic acute kidney injury. Nat Rev Nephrol 2011;7:189-200.

11 Banaei S: Novel role of microRNAs in renal ischemia reperfusion injury. Ren Fail 2015;37:1073-1079.

12 Havasi A, Borkan SC: Apoptosis and acute kidney injury. Kidney Int 2011;80:29-40.

13 Kalogeris T, Baines CP, Krenz M, Korthuis RJ: Cell biology of ischemia/reperfusion injury. Int Rev Cell Mol Biol 2012;298:229-317.

14 Linkermann A, Chen G, Dong G, Kunzendorf U, Krautwald S, Dong Z: Regulated cell death in AKI. J Am Soc Nephrol 2014;25:2689-2701.

15 Bengatta S, Arnould C, Letavernier E, Monge M, de Preneuf HM, Werb Z, Ronco P, Lelongt B: MMP9 and SCF protect from apoptosis in acute kidney injury. J Am Soc Nephrol 2009;20:787-797.

16 Zhang F, Zhao X, Shen X, Zhang C: Molecular mechanisms of cell death in intervertebral disc degeneration. Int J Mol Med 2016;37:1439-1448.

17 Gomes AQ, Nolasco S, Soares H: Non-coding RNAs: multi-tasking molecules in the cell. Int J Mol Sci 2013;14:16010-16039.

18 Yang JX, Rastetter RH, Wilhelm D: Non-coding RNAs: An Introduction. Adv Exp Med Biol 2016;886:13-32.

19 Zhang F, Zhang L, Zhang C: Long noncoding RNAs and tumorigenesis: genetic associations, molecular mechanisms, and therapeutic strategies. Tumour Biol 2016;37:163-175.

20 Esteller M: Non-coding RNAs in human disease. Nat Rev Genet 2011;12:861-874.

21 Sana J, Faltejskova P, Svoboda M, Slaby O: Novel classes of non-coding RNAs and cancer. J Transl Med 2012;10:103.

22 Ma L, Bajic VB, Zhang Z: On the classification of long non-coding RNAs. RNA Biol 2013;10:925-933. 


\section{Kidney \\ Blood Pressure Research}

23 Dozmorov MG, Giles CB, Koelsch KA, Wren JD: Systematic classification of non-coding RNAs by epigenomic similarity. BMC Bioinformatics 2013;14:S2.

24 Li M, Marin-Muller C, Bharadwaj U, Chow KH, Yao Q, Chen C: MicroRNAs: control and loss of control in human physiology and disease. World J Surg 2009;33:667-684.

25 Ha TY: MicroRNAs in Human Diseases: From Cancer to Cardiovascular Disease. Immune Netw 2011;11:135-154.

26 Ardekani AM, Naeini MM: The Role of MicroRNAs in Human Diseases. Avicenna J Med Biotechnol 2010;2:161-179.

27 Croce CM: Causes and consequences of microRNA dysregulation in cancer. Nat Rev Genet 2009;10:704714.

28 Macfarlane LA, Murphy PR: MicroRNA: Biogenesis, Function and Role in Cancer. Curr Genomics 2010;11:537-561.

29 Sharma G, Dua P, Agarwal SM: A Comprehensive Review of Dysregulated miRNAs Involved in Cervical Cancer. Curr Genomics 2014;15:310-323.

30 Li J, Xuan Z, Liu C: Long non-coding RNAs and complex human diseases. Int J Mol Sci 2013;14:1879018808.

31 Wei Q Mi QS, Dong Z: The regulation and function of microRNAs in kidney diseases. IUBMB Life 2013;65:602-614.

32 Cui R, Xu J, Chen X, Zhu W: Global miRNA expression is temporally correlated with acute kidney injury in mice. PeerJ 2016;4:e1729.

33 Li YF, Jing Y, Hao J, Frankfort NC, Zhou X, Shen B, Liu X, Wang L, Li R: MicroRNA-21 in the pathogenesis of acute kidney injury. Protein Cell 2013;4:813-819.

34 Lorenzen JM, Kaucsar T, Schauerte C, Schmitt R, Rong S, Hubner A, Scherf K, Fiedler J, Martino F, Kumarswamy R, Kolling M, Sorensen I, Hinz H, Heineke J, van Rooij E, Haller H, Thum T: MicroRNA-24 antagonism prevents renal ischemia reperfusion injury. J Am Soc Nephrol 2014;25:2717-2729.

35 Gutierrez-Escolano A, Santacruz-Vazquez E, Gomez-Perez F: Dysregulated microRNAs involved in contrastinduced acute kidney injury in rat and human. Ren Fail 2015;37:1498-1506.

36 Bitzer M, Ben-Dov IZ, Thum T: Microparticles and microRNAs of endothelial progenitor cells ameliorate acute kidney injury. Kidney Int 2012;82:375-377.

37 Aguado-Fraile E, Ramos E, Saenz-Morales D, Conde E, Blanco-Sanchez I, Stamatakis K, del Peso L, Cuppen E, Brune B, Bermejo ML: miR-127 protects proximal tubule cells against ischemia/reperfusion: identification of kinesin family member 3B as miR-127 target. PLoS One 2012;7:e44305.

38 Ranganathan P, Jayakumar C, Tang Y, Park KM, Teoh JP, Su H, Li J, Kim IM, Ramesh G: MicroRNA-150 deletion in mice protects kidney from myocardial infarction-induced acute kidney injury. Am J Physiol Renal Physiol 2015;309:F551-558.

39 Lan YF, Chen HH, Lai PF, Cheng CF, Huang YT, Lee YC, Chen TW, Lin H: MicroRNA-494 reduces ATF3 expression and promotes AKI. J Am Soc Nephrol 2012;23:2012-2023.

40 Bhatt K, Wei Q, Pabla N, Dong G, Mi QS, Liang M, Mei C, Dong Z: MicroRNA-687 Induced by HypoxiaInducible Factor-1 Targets Phosphatase and Tensin Homolog in Renal Ischemia-Reperfusion Injury. J Am Soc Nephrol 2015;26:1588-1596.

41 Fan LH, He L, Cao ZQ, Xiang B, Liu L: Effect of ischemia preconditioning on renal ischemia/reperfusion injury in rats. Int Braz J Urol 2012;38:842-854.

42 Lempiainen J, Finckenberg P, Levijoki J, Mervaala E: AMPK activator AICAR ameliorates ischaemia reperfusion injury in the rat kidney. Br J Pharmacol 2013;166:1905-1915.

43 Godwin JG, Ge X, Stephan K, Jurisch A, Tullius SG, Iacomini J: Identification of a microRNA signature of renal ischemia reperfusion injury. Proc Natl Acad Sci USA 2010;107:14339-14344.

44 Xu X, Kriegel AJ, Jiao X, Liu H, Bai X, Olson J, Liang M, Ding X: miR-21 in ischemia/reperfusion injury: a double-edged sword? Physiol Genomics 2014;46:789-797.

45 Zhou J, Wang KC, Wu W, Subramaniam S, Shyy JY, Chiu JJ, Li JY, Chien S: MicroRNA-21 targets peroxisome proliferators-activated receptor-alpha in an autoregulatory loop to modulate flow-induced endothelial inflammation. Proc Natl Acad Sci USA 2011;108:10355-10360.

46 Jia P, Teng J, Zou J, Fang Y, Zhang X, Bosnjak ZJ, Liang M, Ding X: miR-21 contributes to xenon-conferred amelioration of renal ischemia-reperfusion injury in mice. Anesthesiology 2013;119:621-630.

47 Jia P, Teng J, Zou J, Fang Y, Jiang S, Yu X, Kriegel AJ, Liang M, Ding X: Intermittent exposure to xenon protects against gentamicin-induced nephrotoxicity. PLoS One 2013;8:e64329. 


\section{Kidney \\ Blood Pressure Research}

48 Jia P, Teng J, Zou J, Fang Y, Wu X, Liang M, Ding X: Xenon Protects Against Septic Acute Kidney Injury via miR-21 Target Signaling Pathway. Crit Care Med 2015;43:e250-259.

49 Ho J, Ng KH, Rosen S, Dostal A, Gregory RI, Kreidberg JA: Podocyte-specific loss of functional microRNAs leads to rapid glomerular and tubular injury. J Am Soc Nephrol 2008;19:2069-2075.

50 Shi S, Yu L, Chiu C, Sun Y, Chen J, Khitrov G, Merkenschlager M, Holzman LB, Zhang W, Mundel P, Bottinger EP: Podocyte-selective deletion of dicer induces proteinuria and glomerulosclerosis. J Am Soc Nephrol 2008;19:2159-2169.

51 Xiang Y, Cheng J, Wang D, Hu X, Xie Y, Stitham J, Atteya G, Du J, Tang WH, Lee SH, Leslie K, Spollett G, Liu Z, Herzog E, Herzog RI, Lu J, Martin KA, Hwa J: Hyperglycemia repression of miR-24 coordinately upregulates endothelial cell expression and secretion of von Willebrand factor. Blood 2015;125:3377-3387.

52 Agrawal R, Tran U, Wessely 0: The miR-30 miRNA family regulates Xenopus pronephros development and targets the transcription factor Xlim1/Lhx1. Development 2009;136:3927-3936.

53 Bijkerk R, van Solingen C, de Boer HC, van der Pol P, Khairoun M, de Bruin RG, van Oeveren-Rietdijk AM, Lievers E, Schlagwein N, van Gijlswijk DJ, Roeten MK, Neshati Z, de Vries AA, Rodijk M, Pike-Overzet K, van den Berg YW, van der Veer EP, Versteeg HH, Reinders ME, Staal FJ, van Kooten C, Rabelink TJ, van Zonneveld AJ: Hematopoietic microRNA-126 protects against renal ischemia/reperfusion injury by promoting vascular integrity. J Am Soc Nephrol 2014;25:1710-1722.

54 Bijkerk R, van Solingen C, de Boer HC, de Vries DK, Monge M, van Oeveren-Rietdijk A, van der Veer EP, Schaapherder AF, Rabelink TJ, van Zonneveld AJ: Silencing of miRNA-126 in kidney ischemia reperfusion is associated with elevated SDF-1 levels and mobilization of Sca-1+/Lin- progenitor cells. Microrna 2014;3:144-149.

55 Aguado-Fraile E, Ramos E, Conde E, Rodriguez M, Liano F, Garcia-Bermejo ML: MicroRNAs in the kidney: novel biomarkers of acute kidney injury. Nefrologia 2013;33:826-834.

56 Zhou H, Hasni SA, Perez P, Tandon M, Jang SI, Zheng C, Kopp JB, Austin H, Balow JE, Alevizos I, Illei GG: miR150 promotes renal fibrosis in lupus nephritis by downregulating SOCS1. J Am Soc Nephrol 2013;24:10731087.

57 Chen W, Han C, Zhang J, Song K, Wang Y, Wu T: miR-150 Deficiency Protects against FAS-Induced Acute Liver Injury in Mice through Regulation of AKT. PLoS One 2015;10:e0132734.

58 Wang X, Zhang X, Ren XP, Chen J, Liu H, Yang J, Medvedovic M, Hu Z, Fan GC: MicroRNA-494 targeting both proapoptotic and antiapoptotic proteins protects against ischemia/reperfusion-induced cardiac injury. Circulation 2010;122:1308-1318.

59 Yoshida T, Sugiura H, Mitobe M, Tsuchiya K, Shirota S, Nishimura S, Shiohira S, Ito H, Nobori K, Gullans SR, Akiba T, Nitta K: ATF3 protects against renal ischemia-reperfusion injury. J Am Soc Nephrol 2008;19:217224.

60 Welten SM, Bastiaansen AJ, de Jong RC, de Vries MR, Peters EA, Boonstra MC, Sheikh SP, La Monica N, Kandimalla ER, Quax PH, Nossent AY: Inhibition of 14q32 MicroRNAs miR-329, miR-487b, miR-494, and miR-495 increases neovascularization and blood flow recovery after ischemia. Circ Res 2014;115:696-708.

61 Liu Z, Wang S, Mi QS, Dong Z: MicroRNAs in Pathogenesis of Acute Kidney Injury. Nephron 2016; DOI:10.1159/000446551.

62 Lorenzen JM, Kielstein JT, Hafer C, Gupta SK, Kumpers P, Faulhaber-Walter R, Haller H, Fliser D, Thum T: Circulating miR-210 predicts survival in critically ill patients with acute kidney injury. Clin J Am Soc Nephrol 2011;6:1540-1546.

63 Chen HH, Lan YF, Li HF, Cheng CF, Lai PF, Li WH, Lin H: Urinary miR-16 transactivated by C/EBPbeta reduces kidney function after ischemia/reperfusion-induced injury. Sci Rep 2016;6:27945.

64 Lorenzen JM, Schauerte C, Kielstein JT, Hubner A, Martino F, Fiedler J, Gupta SK, Faulhaber-Walter R, Kumarswamy R, Hafer C, Haller H, Fliser D, Thum T: Circulating long noncoding RNATapSaki is a predictor of mortality in critically ill patients with acute kidney injury. Clin Chem 2015;61:191-201.

65 Chen Z, Jia S, Li D, Cai J, Tu J, Geng B, Guan Y, Cui Q Yang J: Silencing of long noncoding RNA AK139328 attenuates ischemia/reperfusion injury in mouse livers. PLoS One 2013;8:e80817.

66 Yu TM, Palanisamy K, Sun KT, Day YJ, Shu KH, Wang IK, Shyu WC, Chen P, Chen YL, Li CY: RANTES mediates kidney ischemia reperfusion injury through a possible role of HIF-1alpha and LncRNA PRINS. Sci Rep $2016 ; 6: 18424$ 